\title{
Real world aspects of palliative trifluridine plus tiperacil (TAS-102) in refractory metastatic colorectal cancer
}

\author{
Mikael Wallander ${ }^{1}$, Bo Rolander ${ }^{2,3}$, Elisabeth Åvall-Lundqvist ${ }^{4,5}$, Nils O. Elander ${ }^{4,5}$ \\ ${ }^{1}$ Department of Oncology, Ryhov County Hospital, SE-55305 Jönköping, Sweden; ${ }^{2}$ Futurum - Academy for Healthcare, Region Jönköping \\ County, Sweden; ${ }^{3}$ Department of Behavioural Science and Social Work, School of Health and Welfare, Jönköping University, Jönköping, Sweden; \\ ${ }^{4}$ Department of Oncology, ${ }^{5}$ Department of Biomedical and Clinical Sciences, Linköping University, SE-58185 Linköping, Sweden \\ Contributions: (I) Conception and design: M Wallander, E Åvall-Lundqvist, NO Elander; (II) Administrative support: None; (III) Provision of \\ study materials or patients: M Wallander, NO Elander; (IV) Collection and assembly of data: M Wallander, NO Elander; (V) Data analysis and \\ interpretation: All authors; (VI) Manuscript writing: All authors; (VII) Final approval of manuscript: All authors. \\ Correspondence to: Nils O. Elander, MD, PhD. Linköping University, SE-58185 Linköping, Sweden. Email: nils.elander@liu.se.
}

Background: While recent randomised phase III trials show that trifluridine/tiperacil (TAS-102) may prolong life in patients with refractory metastatic colorectal cancer (rmCRC), palliative aspects on its efficacy and tolerability in real world patients need further elucidation.

Methods: A retrospective observational multicentre study was designed, including all patients with rmCRC who received TAS-102 under 2016-2019 in the South East Health Care region of Sweden. 48 patients were identified. Primary outcome was overall survival (OS) and secondary outcomes were progression-free survival (PFS), time to ECOG performance status deterioration (PSD), safety and dose reductions, admission to and duration of access to palliative care, and administration of TAS-102 in the last 30 days before death.

Results: Median OS, PFS, and time to PSD (a proxy for impaired quality of life) from start of TAS102 were 6.4 months (95\% CI: 4.4-8.4), 2.3 months (95\% CI: 1.8-2.7) and 2.5 months (95\% CI: 1.9-3.2), respectively. Following uni- and multivariable regression analyses, the number of previous treatment lines ( $\leq 2$ vs. $\geq 3$ ) was statistically independent for OS (median 7.8 vs. 5.3 months, $\mathrm{P}=0.05$ ), PFS (median 2.4 vs. 1.8 months, $\mathrm{P}=0.03$ ), and time to PSD (median 2.8 vs. 1.8 months, $\mathrm{P}=0.03)$. Thirty-four (71\%) of the patients received reduced doses. The most common grade 3-4 toxicity was neutropenia (39\%). Forty-three (90\%) were admitted to GP or hospital-based home palliative care. Median time for access to any form of palliative care before death was 2.3 (95\% CI: 0.5-3.2) months. Few patients ( $n=3,7 \%)$ received their last dose of TAS102 in their last 30 days of life.

Conclusions: The outcome and tolerability of TAS-102 in rmCRC appear similar in a real-world context and randomised trials. The retrospective design and limited sample size preclude firm conclusions on subgroup analyses, but it appears that the prognosis is slightly better the earlier TAS-102 is introduced. Treatment durations are generally short, and early admission to a palliative care provider is recommended.

Keywords: Trifluridine and tiperacil (TAS-102); palliative care; colorectal neoplasms; chemotherapy

Submitted Jan 20, 2020. Accepted for publication Jun 05, 2020.

doi: 10.21037/jgo-20-43

View this article at: http://dx.doi.org/10.21037/jgo-20-43

\section{Introduction}

Colorectal cancer is the third most common cancer worldwide, affecting approximately 1.8 million individuals and accounting for more than 800,000 deaths annually (1). While total survival has improved over the last decades, metastatic colorectal cancer (mCRC) remains a significant therapeutic challenge, and following progressive disease on standard regimens based on 5-fluorouracil (or its oral prodrug capecitabine), irinotecan, oxaliplatin (alone or in combination with anti-VEGF/EGFR antibodies) (2), the 
options for further disease modifying therapies are limited. Two phase III randomised controlled trials, RECOURSE (3) and TERRA (4), have recently evaluated a combination of trifluridine and tiperacil (TAS-102) in refractory mCRC (rmCRC). Both studies report promising results in 'fit' patients with WHO performance status $0-1$, but it is not clear to what extent these results reflect the outcome in patients treated outside the frame of a controlled trial and/ or patients with poor performance status.

Trifluridine is a nucleoside analogue that is incorporated in DNA, which leads to double-strand breaks and inhibits cell proliferation. Tiperacil is an enzyme inhibitor that inhibits rapid degradation of trifluridine, and it has also antiangiogenic properties (5). In a randomised phase II trial, TAS-102 displayed promising outcomes in refractory colorectal cancer previously treated with at least two different regimens (6). In the subsequent RECOURSE phase III trial, median survival was improved from 5.3 months with placebo to 7.1 months with TAS-102 (3). The TERRA study, including an entirely Asian population, showed a significant albeit limited improvement in median OS of 7.8 vs. 7.1 months favouring TAS-102 (4). Several retrospective observational studies indicate that the outcome is comparable in unselected patients $(7,8)$. TAS-102 was approved by the Swedish regulatory authorities in September 2016 for the treatment of rmCRC following progressive disease on standard therapies.

The present study aimed to evaluate the efficacy and safety of TAS-102 in a real-world population of all patients with rmCRC who received TAS-102 following its introduction in clinical practice. Potentially prognostic factors for overall and progression-free survival, as well as time to performance status deterioration (PSD), a surrogate marker for impaired quality of life, were assessed. In addition, treatment beyond progression, admission to palliative care providers, and the use of chemotherapy near end of life were evaluated. We present the following article in accordance with the STROBE (Strengthening the Reporting of Results of Observational Studies in Epidemiology) reporting checklist. Available at http:// dx.doi.org/10.21037/jgo-20-43.

\section{Methods}

The research was conducted in accordance with the Declaration of Helsinki (as revised in 2013). The study was approved by the Regional Ethics Review Board in Linköping (Dnr 2018/139-31). Given the retrospective design, and the presumed nearly $100 \%$ mortality in this type of cohort, the Ethical Board considered it was not necessary or possible to obtain written informed consent.

\section{Study population}

A retrospective multicentre observational study was conducted in the South East Health Care Region of Sweden, covering all three departments treating $\mathrm{mCRC}$ in the region and including the departments of oncology in Jönköping, Linköping, and Kalmar. There are no non-governmental or alternative providers of oncological treatments in this region, which means that all patients living in the region and fulfilling the inclusion criteria were included in this study. Inclusion criteria were metastatic colorectal cancer (mCRC), refractory disease on previous regimens (lines) of standard chemotherapy (rmCRC), and treatment with at least one dose of TAS-102 between November $1^{\text {st }} 2016$ and February $1^{\text {st }} 2019$. To reflect the real-world situation with patients who may or may not differ from those included in previous phase III trials, both 'fit' patients in performance status (PS) $0-1$ and 'frail' patients in PS $\geq 2$ were included. Chemotherapy for mCRC is only administered at the above mentioned centres, and it is prescribed through the digital software CSAM Cytodose system. All patients in the study were therefore identified by this software.

Medical records were reviewed and data on baseline patient and tumour characteristics were collected. Data were recorded in a study specific case report form and included sex, age, baseline biochemistry, site of tumor, number of metastatic sites, prior systemic regimens, KRAS mutation status, Eastern Cooperative Oncology Group (ECOG) performance status (PS), and adverse events. Based on the available information in the medical records, the severity of adverse events (AEs) was graded using the National Center Institute Common Terminology Criteria for Adverse Events (NCI CTCAE version 4.0). The number of unplanned hospitalisations was recorded. To ensure as consistent and robust recording of data as possible, one researcher (M.W.) performed all data collection including a double check of matching information in the medical records and the CRFs. Any uncertainties were discussed with the main supervisor (N.E.) and resolved with a consensus decision. Patients were followed until $24^{\text {th }}$ of February 2019 or death, whatever occurred first.

Admissions to and access to palliative care were recorded. World Health Organization (WHO) defines palliative care as follows: "Palliative care is an approach that improves the 
quality of life of patients and their families facing the problem associated with life-threatening illness, through the prevention and relief of suffering by means of early identification and impeccable assessment and treatment of pain and other problems, physical, psychosocial and spiritual." (https://www.who.int/ cancer/palliative/definition/en/). In the South East Region of Sweden, palliative care is available either concomitant with chemotherapy or restricted to the end of life situation (depending on the individual needs of the patient) and is managed either by a team led by the general practitioner (GP) or by a specialised hospital-based team. While both forms include multi-professional service with planned and on demand home visits by a physician and/or a nurse or paramedic, the specialised hospital-based team is also able to maintain advanced invasive interventions such as drainage of pleural effusion or ascites, analgetic intrathecal catheters, etc. Dates of inclusion in the respective type of palliative care were recorded.

\section{Treatment and follow up}

In agreement with the RECOURSE study (3), TAS-102 was prescribed at a dose of $35 \mathrm{mg} / \mathrm{m}^{2}$ twice daily, days 1-5 and $8-12$, in 28 days cycles. This schedule was repeated until disease progression, unacceptable toxicity, or PS deterioration (PSD). Given the retrospective realworld design, there were no fixed follow up scans or appointments, but according to the general clinical practice at the participating hospitals, treatment response was routinely evaluated with imaging (computed tomography), blood samples, and clinical evaluation every 2-3 cycles. Dose reductions were allowed at the treating oncologist's discretion.

\section{Outcomes}

Primary endpoint was overall survival (OS), which was defined as time from TAS-102 start until date of death or last follow-up.

Key secondary endpoints were progression free survival (PFS), defined as time from TAS-102 start until radiological or clinical progression or death, safety in terms of NCI CTCAE AEs, as well as time from start of TAS-102 until PSD. PSD was defined as deterioration at least one step from baseline (i.e., from PS 0 to 1,1 to 2, etc.). Patient with missing PS data at baseline were not included in PSD assessment. Proxies of quality of life (QoL) in mCRC in terms of AEs likely to affect QoL and ECOG PS have previously been employed and analysed in the RECOURSE patient population (9). Other secondary outcomes were number of treatment cycles, dose intensity, reason for treatment discontinuation, and use of palliative care.

\section{Statistical analysis}

Patient characteristics and treatment data were reported as frequencies and percentages for categorical variables. For continuous variables, median OS, PFS, and time to PSD for the whole cohort and subgroups were estimated using the Kaplan-Meier method, with log rank testing performed for subgroup comparisons, and median with $95 \%$ confidence interval and range. The subgroups were age $<65$ or $>65$ years, PS $0 v s$. 1-2, localisation of the primary tumour (right $v$ s. left $v s$. rectum), metastases versus no metastases at first diagnosis, number of metastatic sites at start of TAS-102 (1-2 vs. $\geq 3$ metastatic sites), KRAS status, and number of prior regimens ( $\leq 2 v s . \geq 3$ prior palliative lines prior palliative lines), time from diagnosis of metastatic disease until start of TAS-102 treatment ( $<18$ or $>18$ months), level of albumin $(<35$ or $\geq 35 \mathrm{~g} / \mathrm{L})$, and occurrence of neutropenia (grade 0-2 versus grade 3-4 according to CTCAE 4.0, based on neutrophil counts checked 1-3 days before each cycle).

Cox regression analyses (10) were made with three dependent variables and four independent variables, to evaluate hazard ratios for the same subgroups. P values $<0.05$ (two-sided) were considered statistically significant.

All variables were tested for proportional hazard assumption and fulfilled the criterion, except KRAS status which did not fulfill the criterion for Cox regression analysis. KRAS status was therefore included in descriptive but not comparative statistics or subgroup analyses. Observations should be independent, and the hazard ratio should be constant across time; that is, the proportionality of hazards from one case to another should not vary over time. The latter assumption is known as the proportional hazards assumption.

Multivariable regression analysis was subsequently performed, including number of previous lines beside ECOG performance status and metastatic burden (both which were previously shown to affect the outcome of TAS-102) and metastatic status at primary diagnosis (which potentially could affect the number of previous palliative treatment lines administered).

Numbers of subjects with missing data on any parameter are displayed in Table 1 . 
Table 1 Basic characteristics of patients with metastatic colorectal cancer treated with trifluridine/tiperacil (TAS-102) in the South East Health Care region

Characteristic Study population $(\mathrm{N}=48), \mathrm{n}[\%]$

\section{Gender}

Female

Male

Age, median [range], years

Body surface, median [range]

Primary tumour site at diagnosis

$\begin{array}{lc}\text { Right colon } & 12[25] \\ \text { Left colon } & 10[21] \\ \text { Rectum } & 22[46] \\ \text { Multiple primary sites } & 4[8]\end{array}$

Distant metastasis at diagnosis

$\begin{array}{ll}\text { No } & 16[33] \\ \text { Yes } & 32[67]\end{array}$

Performance status (ECOG) at start of TAS-102

$\begin{array}{lc}0 & 13[27] \\ 1 & 32[67] \\ 2 & 2[4] \\ \text { Missing } & 1[2]\end{array}$

Number of metastatic sites at start of TAS-102

$\begin{array}{lc}1 & 7[14] \\ 2 & 21[44] \\ \geq 3 & 20[42]\end{array}$

Metastatic site at start of TAS-102

$\begin{array}{lc}\text { Liver } & 33[69] \\ \text { Lung } & 35[73] \\ \text { Lymph nodes } & 13[27] \\ \text { Bone } & 10[21] \\ \text { Peritoneum } & 6[13] \\ \text { Other } & 14[29]\end{array}$

Number of prior treatment regimens

$\begin{array}{lc}1 & 5[10] \\ 2 & 31[65] \\ 3 & 11[23] \\ \geq 4 & 1[2]\end{array}$

Table 1 (continued)
Table 1 (continued)

\begin{tabular}{lc}
\hline Characteristic & Study population $(\mathrm{N}=48), \mathrm{n}[\%]$ \\
\hline KRAS-status & \\
Wild-type & $17[35]$ \\
Mutant & $29[60]$ \\
Missing & $2[4]$ \\
\hline
\end{tabular}

\section{Results}

Fifty patients with refractory metastatic colorectal cancer (rmCRC) had been prescribed TAS-102 during the study period. Of these, two patients did not receive any treatment due to impaired PS and were excluded from further analyses. The final study population of 48 patients received at least one dose of TAS-102. Baseline characteristics are shown in Table 1. The median age was 65 (range, 38-78 years). The primary tumour site was equally distributed between colon and rectum and 32 of the patients $(67 \%)$ had distant metastases at diagnosis. Following primary treatment, 16 patients were diagnosed with distant metastases. Most tumours ( $\mathrm{n}=29,60 \%)$ were KRAS mutant.

At start of TAS-102 treatment, seven patients (15\%) had one metastatic site, 21 (44\%) patients had 2 metastatic sites, and 20 patients $(42 \%)$ had at least 3 metastatic sites. The most common metastatic site was the lung $(\mathrm{n}=35,73 \%)$ followed by the liver ( $n=33,69 \%)$. Almost all patients $(n=45$, 94\%) had ECOG performance status (PS) 0-1. Thirty-one $(65 \%)$ started TAS-102 as $3^{\text {rd }}$ line and $11(23 \%)$ as $4^{\text {th }}$ line treatment. Median follow-up time for the study population was 6.1 months (95\% CI: 4.5-7.4). At cut-off date, 41 (85\%) of patients were dead. No loss to follow up was identified.

\section{Treatment efficacy in the total cobort and in subgroups of patients}

In the total study cohort, the median OS and median PFS were 6.4 months (95\% CI: 4.4-8.4) and 2.3 months (95\% CI: 1.8-2.7), respectively. Median time to PSD was 2.5 months (95\% CI: 1.9-3.2). Kaplan-Meier survival curves were plotted with regard to OS, PFS, and time to PSD (Figure 1). Log rank testing revealed no significant differences in any of these outcomes with regard to ECOG PS, presence of metastases at primary diagnosis, metastatic burden, age, baseline plasma albumin, localisation of primary tumour, time duration from diagnosis until start of 
TAS-102, or treatment associated neutropenia (Table S1). However, the number of previous treatment lines $(\geq 3 v s . \leq 2)$ was statistically significant for OS, PFS, and time to PSD, with $\leq 2$ previous lines representing the most favourable outcome in all endpoints (Figure 1).

Following multivariable regression analyses, the significant impact of the number of previous treatment lines administered $(\geq 3 v s . \leq 2)$ remained, revealing a worse outcome in terms of OS (HR 2.8; 95\% CI: 1.2-6.5, $\mathrm{P}=0.02$ ), PFS (HR 3.1; 95\% CI: 1.3-7.1, $\mathrm{P}=0.01$ ), and time to PSD (HR 3.3; 95\% CI: 1.3-8.1, $\mathrm{P}=0.01$, Table 2) in patients with $\geq 3$ previous treatment lines. While not significant in the univariate analyses (Table S1), the presence of metastases at date of primary diagnosis and the number of metastatic sites at start of TAS-102 were statistically significant or borderline significant for time to PSD (HR 2.7; $95 \%$ CI: $1.1-6.3, \mathrm{P}=0.03$ ) and OS (HR 1.3; 95\% CI: $1.0-1.8$, $\mathrm{P}=0.05$ ), in the respective multivariable analysis (Table 2).

To rule out any major differences in the subgroups who received $\geq 3$ and $\leq 2$ previous lines of therapy, baseline parameters were assessed and compared in these groups of patients. Apart from localisation of primary tumor, with right sided localization being more common in the $\leq 2$ previous lines subgroup, no significant differences were evident (Table S2).

\section{Treatment intensity and safety}

Table 3 shows key treatment parameters. The median number of treatment cycles was 2 (range, $1-8)$. Ten patients (21\%) received only one cycle of TAS-102 and 38 (79\%) received two or more cycles. The mean TAS-102 starting dose in the total cohort was $89 \%$ and $25(52 \%)$ started at full dose.

Of the patients receiving more than one cycle, 18 (47\%) had to reduce the dosage later on (either from baseline full dose, or a second reduction from baseline reduced dose). The number of patients who received reduced dose at any time point, either from start or later on, was 34 (71\%). The most common reason for dose reduction was haematological toxicity which occurred in 15 of the patients receiving more than one cycle, followed by prior extensive chemotherapy $(\mathrm{n}=9)$, impaired PS $(\mathrm{n}=8)$, and gastrointestinal toxicity $(n=2)$. Dose delays $>7$ days occurred in $21(44 \%)$ of the patients. For those 23 subjects starting at reduced dose, the reasons for dose reduction were as follows: prior extensive chemotherapy $(n=9)$, general health deterioration $(n=8)$, persisting adverse event from previous lines of therapy $(n=2)$, and others/unknown $(\mathrm{n}=4)$.

Thirty-eight patients (79\%) discontinued treatment due to progressive disease and 7 (15\%) due to PSD. Toxicity was the sole reason for treatment discontinuation in only 1 patient (2\%). The median time from last cycle of TAS-102 and death was 3.1 months (95\% CI: 2.2-4.2). Three (7\%) of the patients received treatment during the last 30 days of life.

Table 4 displays all reported adverse events graded according to NCI CTCAE v. 4.0. The most common grade 3-4 toxicity was neutropenia, which occurred in 17 (39\%) patients. Other severe toxicity related events were infection $(n=7,15 \%)$, anemia $(n=4,8 \%)$, fatigue $(n=4,8 \%)$, and gastrointestinal (nausea, vomiting and diarrhea combined, $\mathrm{n}=3,6 \%)$. Febrile neutropenia occurred in 2 patients $(7 \%)$. There were no treatment related deaths. Twenty (42\%) patients had one single event and 2 patients (4\%) had two or more events of unplanned hospitalisation under the treatment course. Twenty-six (54\%) patients had no events of unplanned hospitalisation.

\section{Treatment beyond progression and palliative care admissions}

Treatment beyond progression was initiated in 15 (33\%) of the patients. The most common regimen was one including capecitabine, either as monotherapy or in combination with oxaliplatin or a targeted monoclonal antibody (anti-VEGF or -EGFR). Regorafenib was given to two patients and only one patient was included in a clinical trial (Table S3).

Details on admission to GP and hospital based palliative care are displayed in Table 5. When commencing TAS-102 treatment, $12(25 \%)$ patients already received some type of palliative care, most often by their general practitioner. At the end of follow-up, 43 (90\%) patients received palliative care, most often provided by a specialised hospital based palliative care unit. Median time for receiving any form of palliative care (from admission until death) was 2.3 months (95\% CI: 0.5-3.2) (Table 5).

\section{Discussion}

The present study provides real world population-based data on trifluridine/tiperacil (TAS-102) in patients with refractory metastatic colorectal cancer (rmCRC). Median overall and progression free survival (OS, PFS) from initiation of TAS-102 in the total cohort were 6.4 and 2.3 months, respectively. Severe adverse events were rare, and the far most common cause for treatment discontinuation 

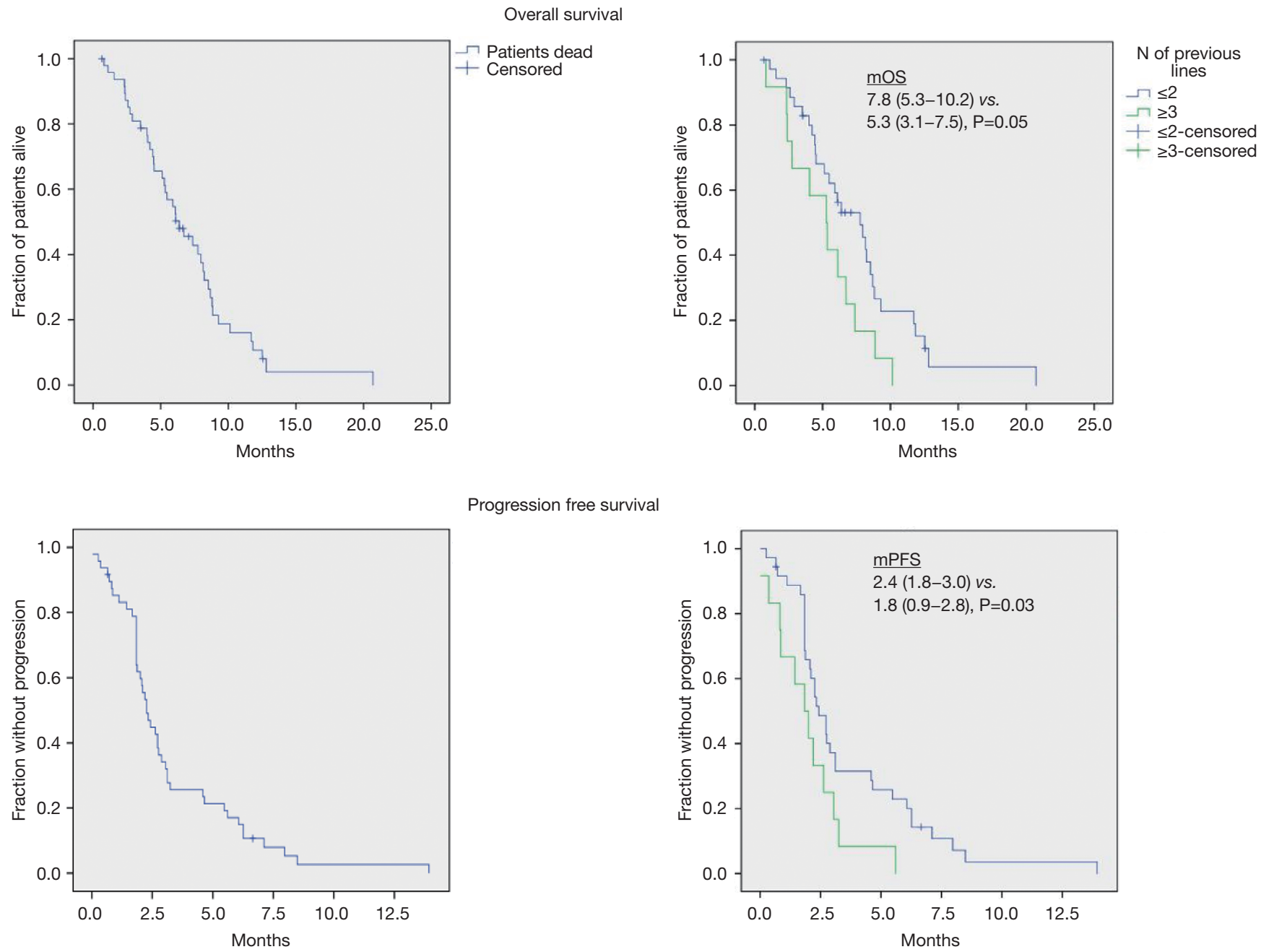

Progression free survival
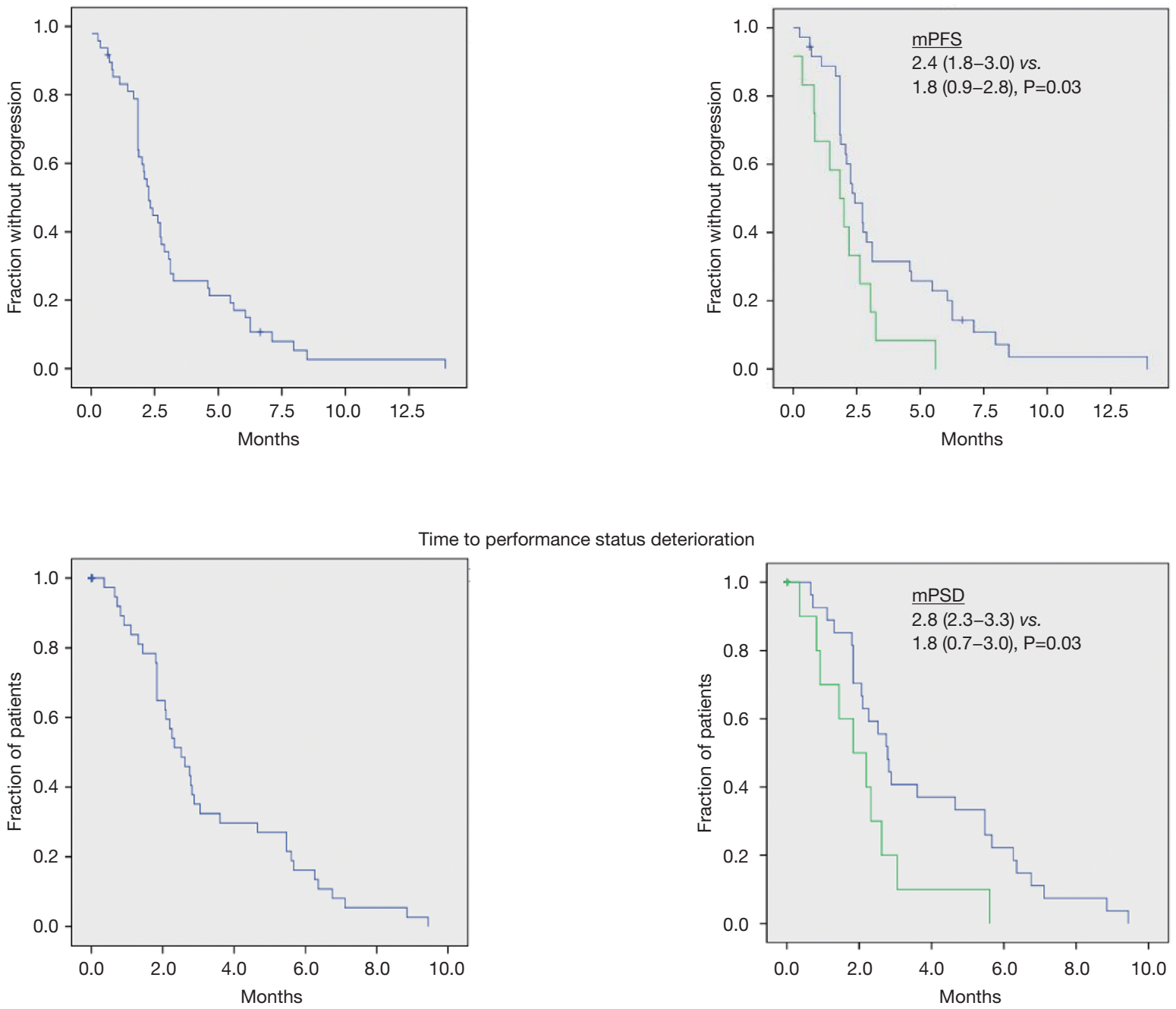

Figure 1 Kaplan-Meier overall survival in total cohort (left column) and by number of previous lines of therapy (right column). Median overall survival (95\% CI) were estimated and log-rank tests were used to compare the differences in survival functions. 
Table 2 Multivariable analysis of overall survival (OS), progression-free survival (PFS), and time to performance status deterioration (PSD)

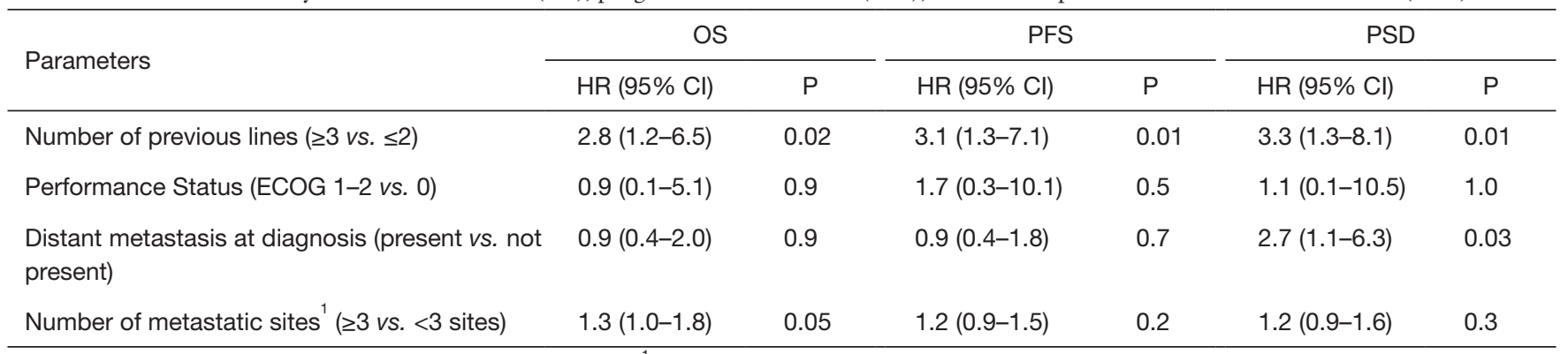

Hazard ratio (HR) estimated by Cox regression analysis. ${ }^{1}$, number of metastatic sites when commencing TAS-102 treatment.

Table 3 Treatment parameters in 48 patients with metastatic colorectal cancer treated with at least one cycle of trifluridine/ tiperacil (TAS-102)

\begin{tabular}{|c|c|}
\hline Parameters & $\begin{array}{l}\mathrm{N} \text { patients [\%] if not } \\
\text { otherwise stated }\end{array}$ \\
\hline $\begin{array}{l}\text { No. of treatment cycles received median } \\
\text { [range] }\end{array}$ & $2[1-8]$ \\
\hline Full dose administered at cycle 1 & $25[52]$ \\
\hline Mean dose administered at cycle $1, \%$ & 89 \\
\hline \multicolumn{2}{|l|}{ Dose reduction } \\
\hline No & 14 [29] \\
\hline Yes, from cycle 1 & $23[48]$ \\
\hline Yes, any cycle & 34 [71] \\
\hline \multicolumn{2}{|l|}{ Reason for dose reduction } \\
\hline Impaired performance status & $8[35]$ \\
\hline Prior extensive chemotherapy & 9 [39] \\
\hline Haematologic toxicity & 15 [83] \\
\hline \multicolumn{2}{|l|}{ Number of treatment delays over 7 days } \\
\hline 0 & $27[56]$ \\
\hline 1 & $13[27]$ \\
\hline 2 & $6[13]$ \\
\hline 3 & $2[4]$ \\
\hline \multicolumn{2}{|l|}{ Reason for treatment discontinuation ${ }^{1}$} \\
\hline Progression & 38 [79] \\
\hline Impaired performance status & $7[15]$ \\
\hline Toxicity & 1 [2] \\
\hline $\begin{array}{l}\text { Time between last cycle of TAS-102 } \\
\text { and death, months, median }[95 \% \mathrm{Cl}]\end{array}$ & $3.1[2.2-4.2]$ \\
\hline $\begin{array}{l}\text { Last dose of TAS-102 during last } \\
30 \text { days of life }\end{array}$ & $3[7]$ \\
\hline
\end{tabular}

Table 4 Adverse events (AEs) estimated by CTCAE v4

\begin{tabular}{|c|c|c|c|c|}
\hline \multirow{2}{*}{$\mathrm{AE}$} & \multicolumn{4}{|c|}{ CTCAE v4 grade, $\mathrm{n}$ [\%] } \\
\hline & 1 & 2 & 3 & 4 \\
\hline Anemia & $26[54]$ & 17 [35] & $4[8]$ & 0 \\
\hline Leukopenia $^{1}$ & $30[64]$ & 0 & 0 & 0 \\
\hline Neutropenia $^{1}$ & $1[2]$ & $7[16]$ & 10 [23] & $7[16]$ \\
\hline Febrile neutropenia & - & - & $2[4]$ & 0 \\
\hline Thrombocytopenia $^{1}$ & $10[22]$ & 0 & 0 & 0 \\
\hline Infection & $3[6]$ & $4[8]$ & 6 [13] & $1[2]$ \\
\hline Gastrointestinal $^{2}$ & 9 [19] & $8[17]$ & $3[6]$ & 0 \\
\hline Skin & $3[6]$ & 0 & 0 & 0 \\
\hline Mucous membrane & $3[6]$ & 0 & 0 & 0 \\
\hline Fatigue & $2[4]$ & $6[13]$ & $4[8]$ & 0 \\
\hline Lung & $1[2]$ & 0 & 0 & 0 \\
\hline Fever & $2[4]$ & & & \\
\hline $\begin{array}{l}1 \text {, information on } \\
47 \text { patients, for neut } \\
48 \text { patients. }{ }^{2} \text {, nause } \\
\text { v4, Common Termin } \\
\text { version } 4.0 .\end{array}$ & $\begin{array}{l}\text { ood cou } \\
\text { ophils in } \\
\text {, vomitins } \\
\text { logy Crit }\end{array}$ & $\begin{array}{l}\text { for leu } \\
\text { and for } \\
\text { and diarrh } \\
\text { a for Ad }\end{array}$ & $\begin{array}{l}\text { cytes a } \\
\text { atelets } \mathrm{i} \\
\text { combin } \\
\text { se Even }\end{array}$ & $\begin{array}{l}\text { lable in } \\
6 \text { out of } \\
\text { CTCAE } \\
\text { CTCAE) }\end{array}$ \\
\hline
\end{tabular}

was disease progression. The number of previous regimens prior to TAS-102 was significantly associated with OS, PFS, and time to performance status deterioration (PSD), with $\leq 2$ previous lines representing a more favourable outcome. In addition, the number of metastatic sites at start of TAS-102 was negatively prognostic for OS, and the presence of distant metastases at diagnosis was negatively prognostic for PSD. While median time from last cycle of TAS-102 to death was limited to 3 months, few patients were treated during the last 30 days of life.

In a recently published systematic review by Andersen 
Table 5 Access to palliative supportive care

\begin{tabular}{lc}
\hline Parameter & $\begin{array}{c}\text { N [\%] if not } \\
\text { otherwise stated }\end{array}$ \\
\hline Palliative supportive care at start of TAS-102 & $12[25]$ \\
GP-based & $7[58]$ \\
Hospital-based & $5[42]$ \\
Palliative supportive care at any time & $43[90]$ \\
GP based & $13[27]$ \\
Hospital based & $30[63]$ \\
Median time months for inclusion $(95 \% \mathrm{Cl})$ & $2.3(0.5-3.2)$ \\
\hline
\end{tabular}

et al. (7), several observational studies with TAS-102 were identified. Whereas time to PSD was not recorded in the Andersen publication, the pooled median OS and median PFS were 6.6 months (95\% CI: 6.1-7.1) and 2.2 months (95\% CI: 2.1-2.3) respectively, which are in line with the results of our study.

In exploratory multivariable regression analyses of potentially prognostic factors, the number of previous regimens prior to TAS-102 were found to be significantly associated with OS, PFS, and PSD. No significant differences in baseline characteristics were evident in patients who had received $\leq 2$ and $\geq 3$ previous lines of therapy, apart from localisation of primary tumour; right sided localisation was more common in the $\leq 2$ lines subgroup. This result should be interpreted with care given the small sample size and, in addition, is unlikely to explain the survival advantage observed since right sidedness is a known to be associated with worse (not better) prognosis (11).

The results on the number of previous lines of therapy are in consistence with the results from a retrospective study by Kwakman et al., including 136 patients from 17 centers in the Netherlands (8). Although details on number of previous regimens prior to TAS-102 were not reported in the Kwakman publication, patients exposed to all standard treatments had a worse outcome compared to those not exposed to all standard regimens, which reasonably should be proportional to the number of previous lines of therapy. Further, in the Kwakman study patients with PS equal to 0 and KRAS wild type were reported to have improved survival. In the present study, PS was not significant with regard to any endpoint, and KRAS was not feasible to analyse since this parameter did not fulfill the criteria for Cox regression analysis.
The results by us and Kwakman collectively suggest that the earlier TAS-102 is introduced, the better is the outcome. Whether this is drug specific, a consequence of acquired multiresistance to anticancer drugs, or merely reflects the natural course of the disease, remains to be elucidated. Of considerable relevance for this matter, the ongoing SOLSTICE phase III trial is now comparing TAS-102 plus bevacizumab versus capecitabine plus bevacizumab as first line treatment for patients not suitable for intensive therapy (12).

In the RECOURSE registration study from 2015 (3), three other factors were identified as prognostic in multivariate Cox regression analysis; time since diagnosis of first metastasis $<18$ or $\geq 18$ months, baseline PS, and number of metastatic sites. In the final subgroup analysis of the RECOURSE Trial (13), primary tumour site (colon vs rectum) was also identified as a prognostic risk factor. While the number of metastatic sites was borderline significant in multivariate analysis on OS, none of the other factors in the RECOURSE trial were statistically significant for primary and key secondary outcomes in our study.

Regarding safety, 39\% of the patients in our study developed neutropenia grade $3-4$, which is comparable with other studies. Kasi et al. (14) and Hamauchi et al. (15) reported neutropenia as a prognostic factor and hypothesised neutropenia as a surrogate marker for adequate antitumor doses. Similarly, in a retrospective study by Cremolini et al. (16), a significant association of grade $\geq 3$ neutropenia with improved PFS and OS was observed. However, in our study no differences were observed when comparing neutropenia grade 0-2 versus grade 3-4 according to CTCAE. While $15 \%$ of the patients were reported to have at least one episode of antibiotic treated infection, febrile neutropenia was registered in only 2 patients (4\%) which is equal to the incidence reported in the RECOURSE trial (3).

While the supplier recommends starting with full dose, a substantial proportion of patients in this study-initiated treatment with dose reduction, and together with dose reductions occurring later on a majority of patients (71\%) received less than full intensity treatment. These findings, together with the present results on OS and PFS, indicate that a slight reduction of the dose might be more feasible in a real-world context and still equally potent as full intensity treatment.

Since this was a retrospective real-world study, and systematic assessment of quality of life was not clinical routine, median time to PSD was considered a surrogate 
marker for impaired quality of life (9). The median time to worsening of PSD was 2.5 months (95\% CI: 1.9-3.2) which almost equals the present PFS data, and PSD was the second most common cause for treatment termination. Median number of cycles were two, which means that the majority of patients will only receive treatment for a limited period of time before termination due to progressive disease and/or impaired general well-being. Consistent with these findings, median time for admission to a palliative care provider was 2.3 months, and by the end of follow up, $90 \%$ of the patients received home based palliative care. Median time from last dose of TAS-102 until date of death was approximately 3 months and few patients received TAS-102 during their last 30 days of life, the latter often being considered a quality indicator of the palliative management in end of life care (17).

The main strength of the present study is the population based real world approach, which means that all patients regardless of, e.g., age, socioeconomic status, comorbidities and performance status were included. The Swedish public funded health care system ensures that all citizens are offered the same type of cancer treatments and access to palliative care. There are no non-governmental health care providers offering oncological treatments in the South East Region of Sweden, which means that all patients living in the area who received TAS-102 and fulfilled the inclusion criteria were included. To our knowledge, the present study provides the hitherto most detailed description about 'soft' parameters such as time to PSD, treatment tolerability, access to palliative care, and proportion of patients receiving treatment during their last month in life in a realworld context. Given the short-expected survival with or without treatment in this type of patients, we believe that these kinds of parameters may be equally or even more important to consider than modest improvements in overall survival.

Naturally, the present study has some limitations, mainly in terms of the limited number of patients and the retrospective design. This means that subgroup analyses should be considered exploratory and the results thereof should be interpreted with care. The lack of control group makes firm conclusions on drug-disease effects difficult. The present data should therefore be considered valuable real-world complements to prospective controlled trials.

\section{Conclusions}

The present study confirms that TAS-102 is a reasonably well tolerated option in a real-world context of refractory mCRC. Survival is however strictly limited, and the treating physician should be observant on early signs of progression and/or impaired performance status, in order to terminate the treatment and/or to admit the patient to a palliative care provider. Subgroup analyses indicate that the prognosis is somewhat better if TAS-102 is given in the third line (or earlier) than in fourth line or later, but these results should be interpreted with care given the limited sample size and retrospective study design.

\section{Acknowledgments}

The authors wish to express their sincere gratitude to Dr Emma Gränsmark, Dr Thomas Blystad, and Dr Hakon Blomstrand, who all provided inspiration and valuable advice, as well as to all clinicians involved in the management of the patients.

Funding: This work was supported by ALF grants Region Östergötland (LIO-697991, LIO- 707011, and LIO697461), CKOC grants Region Östergötland, and the Department of Oncology at Ryhov County Hospital, Jönköping, Sweden.

\section{Footnote}

Reporting Checklist: The authors have completed the STROBE reporting checklist. Available at http://dx.doi. org/10.21037/jgo-20-43

Conflicts of Interest: All authors have completed the ICMJE uniform disclosure form (available at: http://dx.doi. org/10.21037/jgo-20-43). EAL reports COIs outside the submitted work: Roche, Astra Zeneca, Tesario, Clovis Oncology, and Genmab. The other authors have no conflicts of interest to declare.

Ethical Statement: The authors are accountable for all aspects of the work in ensuring that questions related to the accuracy or integrity of any part of the work are appropriately investigated and resolved. The research was conducted in accordance with the Declaration of Helsinki (as revised in 2013). The study was approved by the Regional Ethics Review Board in Linköping (Dnr 2018/139-31). Given the retrospective design, and the presumed nearly $100 \%$ mortality in this type of cohort, the Ethical Board considered it was not necessary or possible to obtain written informed consent. 
Open Access Statement: This is an Open Access article distributed in accordance with the Creative Commons Attribution-NonCommercial-NoDerivs 4.0 International License (CC BY-NC-ND 4.0), which permits the noncommercial replication and distribution of the article with the strict proviso that no changes or edits are made and the original work is properly cited (including links to both the formal publication through the relevant DOI and the license). See: https://creativecommons.org/licenses/by-nc-nd/4.0/.

\section{References}

1. Bray F, Ferlay J, Soerjomataram I, et al. Global cancer statistics 2018: GLOBOCAN estimates of incidence and mortality worldwide for 36 cancers in 185 countries. CA Cancer J Clin 2018;68:394-424.

2. Van Cutsem E, Cervantes A, Adam R, et al. ESMO consensus guidelines for the management of patients with metastatic colorectal cancer. Ann Oncol 2016;27:1386-422.

3. Mayer RJ, Van Cutsem E, Falcone A, et al. Randomized trial of TAS-102 for refractory metastatic colorectal cancer. N Engl J Med 2015;372:1909-19.

4. Xu J, Kim TW, Shen L, et al. Results of a Randomized, Double-Blind, Placebo-Controlled, Phase III Trial of Trifluridine/Tipiracil (TAS-102) Monotherapy in Asian Patients With Previously Treated Metastatic Colorectal Cancer: The TERRA Study. J Clin Oncol 2018;36:350-8.

5. Emura T, Murakami Y, Nakagawa F, et al. A novel antimetabolite, TAS-102 retains its effect on FU-related resistant cancer cells. Int J Mol Med 2004;13:545-9.

6. Yoshino T, Mizunuma N, Yamazaki K, et al. TAS-102 monotherapy for pretreated metastatic colorectal cancer: a double-blind, randomised, placebo-controlled phase 2 trial. Lancet Oncol 2012;13:993-1001.

7. Andersen SE, Andersen IB, Jensen BV, et al. A systematic review of observational studies of trifluridine/Tiparacil (TAS-102) for metastatic colorectal cancer. Acta Oncologica 2019;58:1149-57.

8. Kwakman JJM, Vink G, Vestjens JH, et al. Feasibility and effectiveness of trifluridine/tipiracil in metastatic colorectal cancer: real-life data from The Netherlands. Int J Clin Oncol 2018;23:482-9.

9. Van Cutsem E, Falcone A, Garcia-Carbonero R, et al. Proxies of quality of life in metastatic colorectal cancer: analyses in the RECOURSE trial. ESMO Open 2017;2:e000261.

10. Cox DR. Regression models and life-tables. J R Stat Soc B 1972;34:187-220.

11. Yahagi M, Okabayashi K, Hasegawa H, et al. The Worse Prognosis of Right-Sided Compared with Left-Sided Colon Cancers: a Systematic Review and Meta-analysis. J Gastrointest Surg 2016;20:648-655.

12. André T, Saunders M, Kanehisa A, et al. First-line trifluridine/tipiracil plus bevacizumab for unresectable metastatic colorectal cancer: SOLSTICE study design. Future Oncol 2020;16:21-9.

13. Van Cutsem E, Mayer RJ, Laurent S, et al. The subgroups of the phase III RECOURSE trial of trifluridine/tipiracil (TAS-102) versus placebo with best supportive care in patients with metastatic colorectal cancer. Eur J Cancer 2018;90:63-72.

14. Kasi PM, Kotani D, Cecchini M, et al. Chemotherapy induced neutropenia at 1-month mark is a predictor of overall survival in patients receiving TAS-102 for refractory metastatic colorectal cancer: a cohort study. BMC Cancer 2016;16:467.

15. Hamauchi S, Yamazaki K, Masuishi T, et al. Neutropenia as a Predictive Factor in Metastatic Colorectal Cancer Treated With TAS-102. Clin Colorectal Cancer 2017;16:51-7.

16. Cremolini C, Rossini D, Martinelli E, et al. Trifluridine/ Tiparacil (TAS-102) in Refractory Metastatic Colorectal Cancer: A Multicenter Register in the Frame of the Italian Compassionate Use Program. Oncologist 2018;23:1178-87.

17. Randén $M$, Helde-Frankling $M$, Runesdotter $S$, et al. Treatment decisions and discontinuation of palliative chemotherapy near the end-of-life, in relation to socioeconomic variables. Acta Oncologica 2013;52:1062-6.
Cite this article as: Wallander M, Rolander B, Åvall-Lundqvist E, Elander NO. Real world aspects of palliative trifluridine plus tiperacil (TAS-102) in refractory metastatic colorectal cancer. J Gastrointest Oncol 2020;11(4):616-625. doi: 10.21037/jgo-20-43 
Supplementary

Table S1 Log rank P values for subgroup analyses

\begin{tabular}{llc}
\hline \multirow{2}{*}{ Subgroups } & \multicolumn{2}{c}{ Log rank P value } \\
\cline { 2 - 3 } & OS PFS & 0.033 \\
Previous lines ( $\geq 3$ vs. $\leq 2)$ & 0.050 & 0.033 \\
Age (<65 vs. $>65$ years) & 0.603 & 0.239 \\
Metastatic disease at primary diagnosis (yes vs. no) & 0.879 & 0.972 \\
Metastatic sites ( $\geq 3$ vs. $\leq 2)$ & 0.143 & 0.115 \\
ECOG PS (2 vs. 0-1) & 0.077 & 0.143 \\
Time from primary diagnosis until start of TAS-102 (18 months) & 0.630 & 0.242 \\
Plasma albumin (<35 vs. $\geq 35)$ & 0.087 & 0.150 \\
Neutropenia (3-4 vs. 0-2 CTCAEv4.0) & 0.525 & 0.035 \\
\hline
\end{tabular}


Table S2 Baseline characteristics of patients who received $\leq 2 v s . \geq 3$ lines of therapy before TAS-102

\begin{tabular}{|c|c|c|c|}
\hline Characteristic & $\leq 2$ prev. lines & $\geq 3$ prev. lines & $P$ value \\
\hline Gender & & & 0.311 \\
\hline Female & $17[47]$ & $3[25]$ & \\
\hline Male & 19 [53] & $9[75]$ & \\
\hline Age & & & 0.318 \\
\hline$<65$ years & $20[56]$ & $4[33]$ & \\
\hline$>65$ years & $16[44]$ & $8[67]$ & \\
\hline Body surface, median (range) & $1.97(1.43-2.25)$ & $1.89(1.55-2.25)$ & 0.711 \\
\hline Primary tumour site at diagnosis & & & 0.02 \\
\hline Right colon & $11[31]$ & $1[8]$ & \\
\hline Left colon & $4[11]$ & $6[50]$ & \\
\hline Rectum & $17[47]$ & $5[42]$ & \\
\hline Multiple primary sites & $4[11]$ & $0[0]$ & \\
\hline Distant metastasis at diagnosis & & & 0.073 \\
\hline No & 9 [25] & $7[58]$ & \\
\hline Yes & 27 [75] & $5[42]$ & \\
\hline Performance status (ECOG) at start of TAS-102 & & & 0.464 \\
\hline 0 & $11[31]$ & $2[17]$ & \\
\hline $1-2$ & 24 [69] & 10 [83] & \\
\hline Number of metastatic sites at start of TAS-102 & & & 0.198 \\
\hline $1-2$ & $23[64]$ & $5[42]$ & \\
\hline$\geq 3$ & $13[36]$ & $7[58]$ & \\
\hline \multicolumn{4}{|l|}{ Metastatic site at start of TAS- 102} \\
\hline Liver & & & 1 \\
\hline Yes & 25 [69] & $8[67]$ & \\
\hline No & $11[31]$ & $4[33]$ & \\
\hline Lung & & & 0.469 \\
\hline Yes & 25 [69] & 10 [83] & \\
\hline No & $11[31]$ & $2[17]$ & \\
\hline Lymph nodes & & & 0.469 \\
\hline Yes & $11[31]$ & $2[17]$ & \\
\hline No & 25 [69] & 10 [83] & \\
\hline Bone & & & 0.695 \\
\hline Yes & $7[19]$ & $3[25]$ & \\
\hline No & 29 [81] & $9[75]$ & \\
\hline Peritoneum & & & 0.156 \\
\hline Yes & $3[8]$ & $3[25]$ & \\
\hline No & 33 [92] & 9 [75] & \\
\hline Other & & & 0.726 \\
\hline Yes & $10[28]$ & $4[33]$ & \\
\hline No & 26 [72] & $8[67]$ & \\
\hline KRAS-status* & & & 1 \\
\hline Wild-type & $13[38]$ & 4 [33] & \\
\hline Mutant & 21 [62] & $8[67]$ & \\
\hline
\end{tabular}

Numbers and percentages are given (\%). Chi-square test was performed in all analyses, where Fischer's exact test was reported, except in the continuous variable 'body surface area'. In this analysis independent samples $t$-test was performed. *, missing 2 [4]. 
Table S3 Treatment beyond progression on TAS-102

\begin{tabular}{lc}
\hline Further treatment & $\mathrm{N}$ \\
\hline Total & $15(33 \%)$ \\
Capecitabine & 2 \\
Capecitabine + anti-VEGF antibody & 2 \\
Capecitabine + anti-EGFR antibody & 1 \\
Capecitabine + oxaliplatin & 1 \\
Capecitabine + oxaliplatin + anti-VEGF & 1 \\
5FU + oxaliplatin & 1 \\
Irinotecan & 1 \\
Tegafur + oxaliplatin & 1 \\
Tegafur + oxaliplatin + anti-VEGF & 1 \\
Regorafenib & 2 \\
Anti-EGFR monotherapy & 1 \\
Study drug & 1 \\
\hline
\end{tabular}

